\title{
Primary Electron Transfer Reactions in Membrane-Bound Open and Closed Reaction Centers from Purple Bacterium Rhodobacter sphaeroides
}

\author{
K. Gibasiewicz ${ }^{a, *}$, M. Pajzderska $^{a}$, J. KarolczaK $^{a}$, G. Burdziński $^{a}$, A. DobeK $^{a}$ \\ AND M.R. JONES ${ }^{b}$ \\ ${ }^{a}$ Department of Physics, Adam Mickiewicz University, Umultowska 85, 61-614 Poznań, Poland \\ ${ }^{b}$ School of Biochemistry, Medical Sciences Building, University of Bristol, University Walk, \\ Bristol, BS8 1TD, United Kingdom
}

\begin{abstract}
Most ultrafast transient absorption studies of primary electron transfer in reaction centers from purple bacteria have been performed in complexes isolated from their natural lipid membrane environment using detergent. In this contribution we present near-UV-vis transient absorption studies of reaction centers embedded in their natural membrane environment. The evolution of absorption spectra recorded with subpicosecond resolution and reflecting primarily electron transfer reactions has been compared to data obtained previously for isolated reaction centers. We conclude that the overall spectral evolution in both types of samples is similar, and the environment of the reaction center protein has only a minor effect on the primary electron transfer reactions. The differences between the two samples are explained in terms of different energetic levels (and their different temporal evolution) of the two initial charge separated states $\mathrm{P}^{+} \mathrm{B}_{\mathrm{A}}^{-}$and $\mathrm{P}^{+} \mathrm{H}_{\mathrm{A}}^{-}$, with $\mathrm{P}$ being the primary electron donor and $\mathrm{B}_{\mathrm{A}}$ and $\mathrm{H}_{\mathrm{A}}$ the two consecutive electron acceptors. Additionally, in the electric field generated by $\mathrm{P}^{+} \mathrm{H}_{\mathrm{A}}^{-}$, $\mathrm{B}_{\mathrm{A}}$ in membrane-bound reaction centers undergoes a stronger electrochromic shift than in isolated reaction centers.
\end{abstract}

PACS: 82.50.-m, 87.64.K-, 87.15.M-

\section{Introduction}

Photosynthetic reaction centers (RCs) are pigmentprotein complexes embedded in a lipid bilayer which convert the energy of electronic excitation into the energy of charge separated states. This conversion is preceded by absorption of light by the pigment molecules of the $\mathrm{RC}$ or, more commonly, of additional antenna pigment-protein complexes whose main function is to absorb light and transfer its energy to the RC. In order to study the electron transfer (ET) reactions in RCs by optical methods it is convenient to remove the antenna system, the signals of which overlap spectrally and kinetically with the signals from the RC. One way to do this is by solubilizing the membranes with detergent and then separating the RCs from the antenna complexes by biochemical techniques, a procedure which involves replacement of the natural lipid environment of the $\mathrm{RC}$ with detergent. In this contribution we have investigated membrane-bound RCs (mbRCs) which are RCs embedded in natural membrane from which the antenna pigment-proteins are absent through gene deletions $[1,2]$.

\footnotetext{
* corresponding author; e-mail: krzyszgi@amu.edu.pl
}

The ET cofactors of the RC from the purple bacterium Rhodobacter sphaeroides and their absorbance spectra are presented in Fig. $1[3,4] . \mathrm{P}_{\mathrm{A}}$ and $\mathrm{P}_{\mathrm{B}}$ are two bacteriochlorophyll (BChl) molecules forming a dimer that is a primary electron donor, $\mathrm{P}$ (Fig. 1A). The $\mathrm{BChl}_{\mathrm{A}}$, bacteriopheophytin (BPhe) $\mathrm{H}_{\mathrm{A}}$, and quinones $\mathrm{Q}_{\mathrm{A}}$ and $\mathrm{Q}_{\mathrm{B}}$ are consecutive electron transfer acceptors (route of electron transfer is shown by the arrows in Fig. 1A), whereas the $\mathrm{B}_{\mathrm{B}} \mathrm{BChl}$ and $\mathrm{H}_{\mathrm{B}}$ BPhe are essentially inactive in ET. Consecutive ET steps leading to formation of the radical pair states $\mathrm{P}^{+} \mathrm{H}_{\mathrm{A}}^{-}(\approx 3-6$ ps; charge separation $)$ and $\mathrm{P}^{+} \mathrm{Q}_{\mathrm{A}}^{-}(\approx 200-250 \mathrm{ps}$; charge stabilization $)$ are very well characterized spectrally and kinetically in isolated RCs, but it is difficult to observe experimentally the state $\mathrm{P}^{+} \mathrm{B}_{\mathrm{A}}^{-}$since its decay is faster than its formation. However, it was postulated that there is an observable equilibrium between the states $\mathrm{P}^{+} \mathrm{B}_{\mathrm{A}}^{-}$and $\mathrm{P}^{+} \mathrm{H}_{\mathrm{A}}^{-}$ during the whole lifetime of the state $\mathrm{P}^{+} \mathrm{H}_{\mathrm{A}}^{-}$, usually significantly shifted towards the latter species $[5,6]$. The ET reactions in $\mathrm{RCs}$ may be summarized

$$
\mathrm{P}^{*} \rightarrow \mathrm{P}^{+} \mathrm{B}_{\mathrm{A}}^{-} \leftrightarrow \mathrm{P}^{+} \mathrm{H}_{\mathrm{A}}^{-} \rightarrow \mathrm{P}^{+} \mathrm{Q}_{\mathrm{A}}^{-} \leftrightarrow \mathrm{P}^{+} \mathrm{Q}_{\mathrm{B}}^{-},
$$

where $\mathrm{P}^{*}$ is the first excited singlet state of $\mathrm{P}$. In the notation used above it is assumed that the remaining ET cofactors are in their neutral state, for e.g. 
$\mathrm{P}^{+} \mathrm{H}_{\mathrm{A}}^{-} \equiv \mathrm{P}^{+} \mathrm{B}_{\mathrm{A}} \mathrm{H}_{\mathrm{A}}^{-} \mathrm{Q}_{\mathrm{A}} \mathrm{Q}_{\mathrm{B}}$. The consecutive ET reactions are driven energetically, the free energy of each state being lower than that of the preceding state. Additionally, the energy levels, in particular that of $\mathrm{P}^{+} \mathrm{H}_{\mathrm{A}}^{-}$, may evolve in time due to the dielectric response from the protein to the appearance of charge separated states, a process referred to as relaxation.

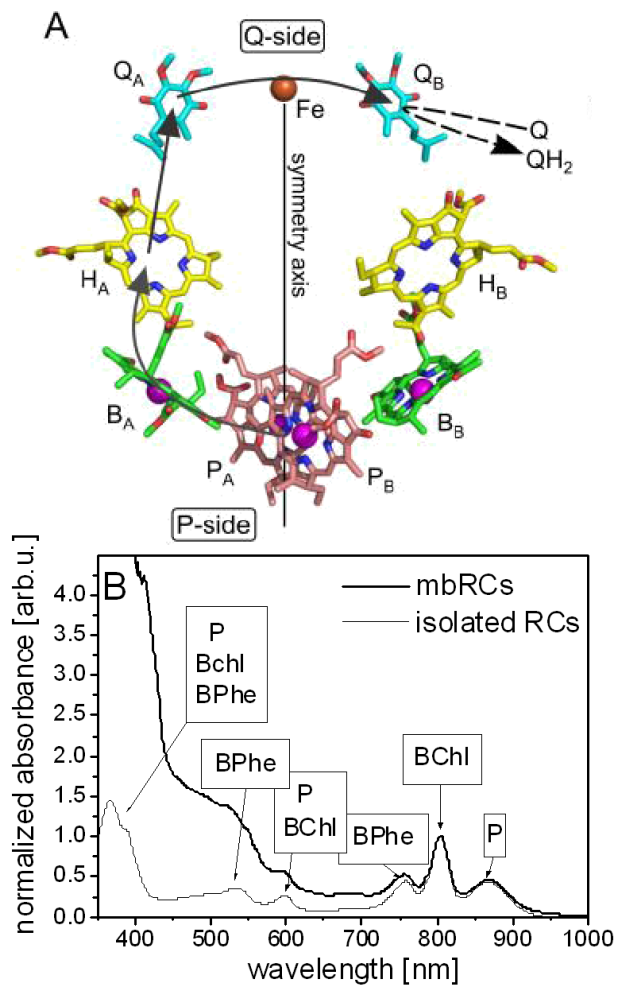

Fig. 1. Structure and stationary absorption spectra of electron transfer cofactors in the RC from $R b a$. sphaeroides. (A) Arrangement of cofactors in the RC - the direction of light-driven electron transfer is indicated by the arrows. (B) Absorption spectra of the respective preparations of the $\mathrm{RCs}$.

RCs in which ET from $\mathrm{P}$ to the consecutive acceptors is possible are described as "open". However, the ET from $\mathrm{H}_{\mathrm{A}}^{-}$to $\mathrm{Q}_{\mathrm{A}}$ may be blocked by permanent reduction of $\mathrm{Q}_{\mathrm{A}}$, and such RCs are described as being "closed". In closed RCs, charge recombination occurs leading mostly to the ground electronic states of all the cofactors [7]:

$$
\mathrm{P}^{+} \mathrm{B}_{\mathrm{A}}^{-} / \mathrm{P}^{+} \mathrm{H}_{\mathrm{A}}^{-} \rightarrow \mathrm{PB}_{\mathrm{A}} \mathrm{H}_{\mathrm{A}} \text {. }
$$

This charge recombination occurs in a multiexponential fashion caused probably by energetic relaxation of the state $\mathrm{P}^{+} \mathrm{H}_{\mathrm{A}}^{-}[8,9]$, and it is probable that the state $\mathrm{P}^{+} \mathrm{H}_{\mathrm{A}}^{-}$recombines mainly via the thermally activated state $\mathrm{P}^{+} \mathrm{B}_{\mathrm{A}}^{-}[5,6,10]$.

The main goal of the work described in this contribution was to compare ET reactions in mbRCs and in isolated RCs and to elucidate differences between these two types of preparations.

\section{Materials and methods}

Antenna-deficient strains of Rba. sphaeroides were grown under dark/semi-aerobic conditions as described elsewhere [1], and intracytoplasmic membranes containing mbRCs as the sole pigment-protein complex were isolated by breakage of harvested cells in a French pressure cell, followed by purification on $15 \% / 40 \%$ (w/v) sucrose density step gradients [11].

Femtosecond transient absorption pump-probe measurements were performed with $\approx 300$ fs excitation at $800 \mathrm{~nm}$ in $2 \mathrm{~ns}$ temporal window and in 330-700 $\mathrm{nm}$ spectral window as described elsewhere [6]. The mbRCs of $\mathrm{OD}_{800 \mathrm{~nm}, 1 \mathrm{~cm}} \approx 1.8$ were suspended in a buffer comprising Tris- $\mathrm{HCl}$ ( $\mathrm{pH} 8.0$ ) $/ 0.025 \%$ lauryl dimethylamine oxide (LDAO) and placed in a rotating cuvette yielding $a \approx 1.5 \mathrm{~mm}$ optical path of the probe laser beam through the sample. Time-resolved spectra were analyzed using global analysis fitting [12, 13] and the program ASUFIT (available at ww. public.asu.edu/ laserweb/ asufit/asufit.html). In order to close RCs, a weak background illumination in the presence of $10 \mathrm{mM}$ sodium ascorbate was applied [6]. It was additionally necessary to add $10 \mu \mathrm{M} o$-phenanthroline to mbRCs, a herbicide which replaces quinone in site $\mathrm{Q}_{\mathrm{B}}$, as otherwise $\mathrm{Q}_{\mathrm{A}}^{-}$was efficiently reoxidized by $\mathrm{Q}_{\mathrm{B}}$.

\section{Results and discussion}

Figure 1B compares steady-state absorption spectra of mbRCs and isolated RCs. The spectrum of mbRCs is strongly distorted by scattering at shorter wavelengths due to the relatively large size of the membrane particles, making the sample somewhat turbid. As a result the spectrum of the RC cofactors (thin line in Fig. 1B) is superimposed on a baseline that steeply increased with decreasing wavelength. Interestingly, this strong distortion did not affect dramatically the quality of the transient absorption data collected between 330 and $700 \mathrm{~nm}$ (see below).

Figure 2 shows decay associated spectra (DAS) $[12,13]$ obtained in a global analysis of transient absorption spectra for open and closed mbRCs (Fig. 2A and B, respectively) following $\approx 300 \mathrm{fs}$ excitation at $800 \mathrm{~nm}$. These spectra should be directly compared with the respective DAS for isolated RCs which were published previously (Fig. $1 \mathrm{~A}$ and D in Ref. [6]).

For open mbRCs (Fig. 2A), four kinetic components were resolved in the global analysis. The fastest $0.3 \mathrm{ps}$ phase was assigned to energy transfer from the initially excited monomeric BChls $\left(\mathrm{B}_{\mathrm{A}}\right.$ and $\left.\mathrm{B}_{\mathrm{B}}\right)$ to the primary electron donor $\mathrm{P}$ (excitation at $800 \mathrm{~nm}$ leads initially to the formation of (mainly) the excited states of $B_{A}$ and $\mathrm{B}_{\mathrm{B}}$, which transfer their excited state energy to $\mathrm{P}$ ). The two slower phases, of $\approx 5$ and $\approx 240$ ps were assigned to the primary charge separation (electron transfer from $\mathrm{P}^{*}$ to the $\mathrm{BPhe} \mathrm{H}_{\mathrm{A}}$ ) and charge stabilization (electron transfer from $\mathrm{H}_{\mathrm{A}}^{-}$to $\mathrm{Q}_{\mathrm{A}}$ ), respectively, whereas the non-decaying component was assigned to the state 


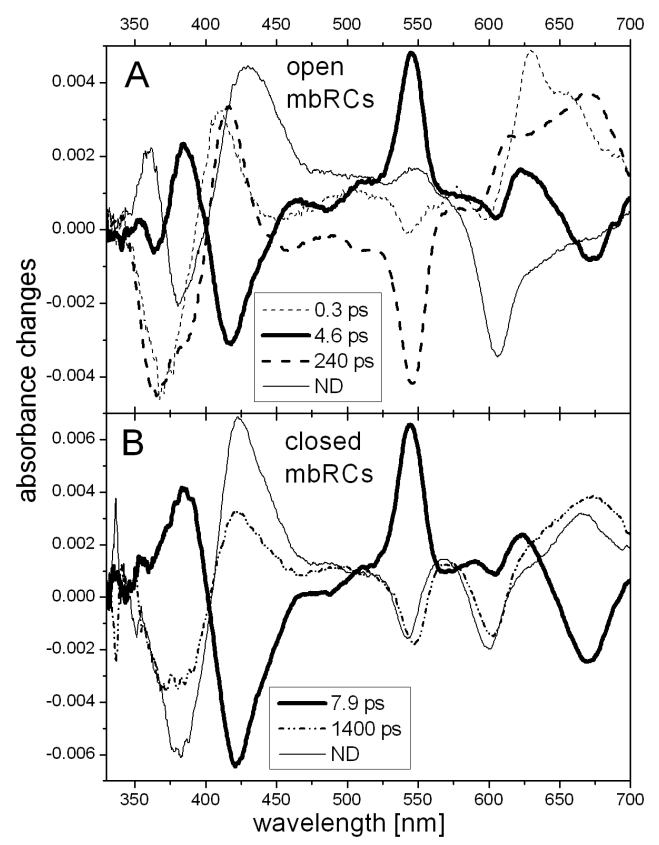

Fig. 2. Decay associated spectra of open and closed membrane-bound RCs from Rba. sphaeroides. (A) Open mbRCs and (B) closed mbRCs.

$\mathrm{P}^{+} \mathrm{Q}_{\mathrm{A}}^{-}$. The lifetimes, lineshapes, and, in consequence, interpretation of the DAS obtained for membrane-bound RCs were very similar to those reported for isolated RCs (Fig. 1A in Ref. [6]). A detailed interpretation of individual transient absorption bands of each DAS was given in previous studies $[6,14]$. For the purposes of the present contribution it is relevant to note that the bands at $\approx 545$ and $\approx 670 \mathrm{~nm}$, in the different DAS in both open and closed mbRCs are attributable to changes in the excitation/redox state of the $\mathrm{BPhe} \mathrm{H}_{\mathrm{A}}$, whereas the band at $\approx 600 \mathrm{~nm}$ is attributable to changes in the state of $\mathrm{P}$ and/or the BChl $\mathrm{B}_{\mathrm{A}}$ (compare with Fig. 1B). In the so-called Soret region (330-450 nm), absorption changes from $\mathrm{P}, \mathrm{B}_{\mathrm{A}}$, and $\mathrm{H}_{\mathrm{A}}$ overlap each other to a higher extent than in so-called $\mathrm{Q}_{\mathrm{X}}$ region $(\approx 540-600 \mathrm{~nm})$. Positive bands in all the DAS reveal reactions during which the absorption of a particular cofactor decreases. These are mainly the decay of stationary absorption (photobleaching), or the decay of the absorption of a transient species such as the excited, reduced or oxidized form of a cofactor. Negative bands reveal reactions during which the absorption of particular cofactors increases (recovery of stationary absorption or the appearance of transient absorption).

DAS for closed mbRCs are presented in Fig. 2B. The fastest, subpicosecond phase, essentially identical to the respective phase in the data for open RCs and assigned to the energy transfer step, has been omitted. In the case of the closed mbRCs it was found that prolonged background illumination of the sample, intended to close RCs, caused incomplete reduction of $\mathrm{Q}_{\mathrm{A}}$. The shapes and lifetimes of the DAS obtained from the measurements under these conditions therefore clearly revealed a presence of a mixture of open and closed RCs in the sample. A way to overcome this difficulty was to shorten the data acquisition, and thus background illumination time. Therefore, the spectra presented in Fig. 2B were constructed on the basis of the measurements performed for only 25 delay times, compared with 70 delay times for open mbRCs (and for both open and closed isolated RCs in previous work [6]). This resulted in $\approx$ threefold shorter experiments and allowed almost all of the mbRCs to be kept in the closed state. The drawback of an experimental procedure employing a smaller number of delay times was a naturally poorer temporal resolution which influenced mainly the "subpicosecond" DAS (not shown) and also to a lesser extent the next DAS attributed to charge separation (7.9 ps, Fig. 1B). On the other hand, the two slowest DAS were fully comparable with the data obtained for open mbRCs (Fig. 2A) and for isolated RCs [6].

We have no definite explanation for the observation of decreasing efficiency of $\mathrm{Q}_{\mathrm{A}}$ reduction under continuous background low illumination. We may only speculate that under prolonged illumination (1) there is some competition between $o$-phenanthroline and quinones in docking to $\mathrm{Q}_{\mathrm{B}}$ site, or (2) another electron acceptor that reoxidizes $\mathrm{Q}_{\mathrm{A}}^{-}$appears, or (3) sodium ascorbate does

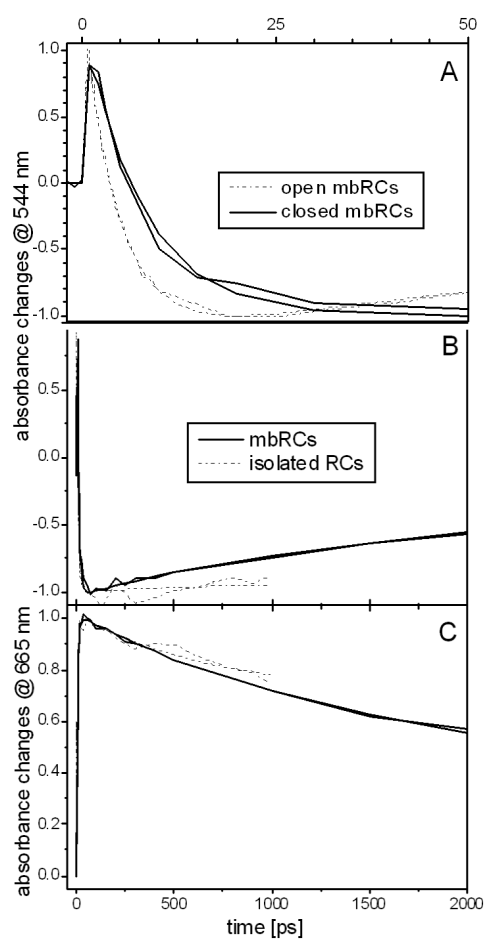

Fig. 3. Transient absorption kinetics of membrane-bound (mbRCs) and isolated RCs from Rba. sphaeroides at two wavelengths characteristic for BPhe $\mathrm{H}_{\mathrm{A}}$. Each experimental trace is accompanied by its fit. (A) Charge separation kinetics monitored at $544 \mathrm{~nm}$ (formation of $\mathrm{H}_{\mathrm{A}}^{-}$) in open and closed mbRCs. (B) and (C) Charge recombination kinetics monitored at $544 \mathrm{~nm}(\mathrm{~B})$ and at $665 \mathrm{~nm}(\mathrm{C})$ (decay of $\mathrm{H}_{\mathrm{A}}^{-}$) in closed mbRCs and isolated RCs. 
not efficiently re-reduce $\mathrm{P}^{+}$(after formation of background light-induced formation of $\mathrm{P}^{+} \mathrm{Q}_{\mathrm{A}}^{-}$) and each act of $\mathrm{P}^{+} \mathrm{Q}_{\mathrm{A}}^{-}$formation is followed by $\mathrm{P}^{+} \mathrm{Q}_{\mathrm{A}}^{-} \rightarrow \mathrm{P}^{+} \mathrm{Q}_{\mathrm{A}}$ charge recombination. The last possibility may happen if $\mathrm{P}^{+} \mathrm{Q}_{\mathrm{A}}^{-} \rightarrow \mathrm{P}^{+} \mathrm{Q}_{\mathrm{A}}$ charge recombination is faster than $\mathrm{P}^{+}$re-reduction by ascorbate.

Despite the poorer temporal resolution in the case of closed mbRCs, the shape of the charge separation DAS (Fig. 2B, thick line) was almost identical to the respective DAS obtained for open mbRCs (Fig. 2A, thick line). On the other hand, the lifetime associated with this DAS increased from $4.6 \mathrm{ps}$ in open mbRCs to $7.9 \mathrm{ps}$ in closed mbRCs. Although the latter value may have been a little overestimated due to the poorer temporal resolution, the increase in the charge separation lifetime was real (see Fig. 3A for comparison of the absorbance changes at
$544 \mathrm{~nm}$, a wavelength characteristic for $\mathrm{H}_{\mathrm{A}}$, in open and closed mbRCs). A similar increase in lifetime for charge separation was observed previously in isolated RCs upon their closure (from 5.2 to 6.4 ps [6]; see also Refs. [15, 16]).

The two slower DAS in closed mbRCs, $1400 \mathrm{ps}$ and non-decaying (ND), were similar in shape to each other and clearly reflect two phases of $\mathrm{P}^{+} \mathrm{H}_{\mathrm{A}}^{-} \rightarrow \mathrm{PH}_{\mathrm{A}}$ charge recombination (Fig. $2 \mathrm{~B}$ ). Negative bands at $\approx 545 \mathrm{~nm}$ (due to the re-oxidation of $\mathrm{H}_{\mathrm{A}}^{-} \rightarrow \mathrm{H}_{\mathrm{A}}$ ) and at $\approx 600 \mathrm{~nm}$ (due to the decay of $\mathrm{P}^{+}$and $\mathrm{B}_{\mathrm{A}}^{-}$species through their re-reduction and re-oxidation, respectively), and also other bands, demonstrate decay of the state $\mathrm{P}^{+} \mathrm{H}_{\mathrm{A}}^{-}$being in equilibrium with the state $\mathrm{P}^{+} \mathrm{B}_{\mathrm{A}}^{-}$(charge recombination). Similar multiexponential charge recombination was reported previously for isolated RCs $[5,6,9]$.

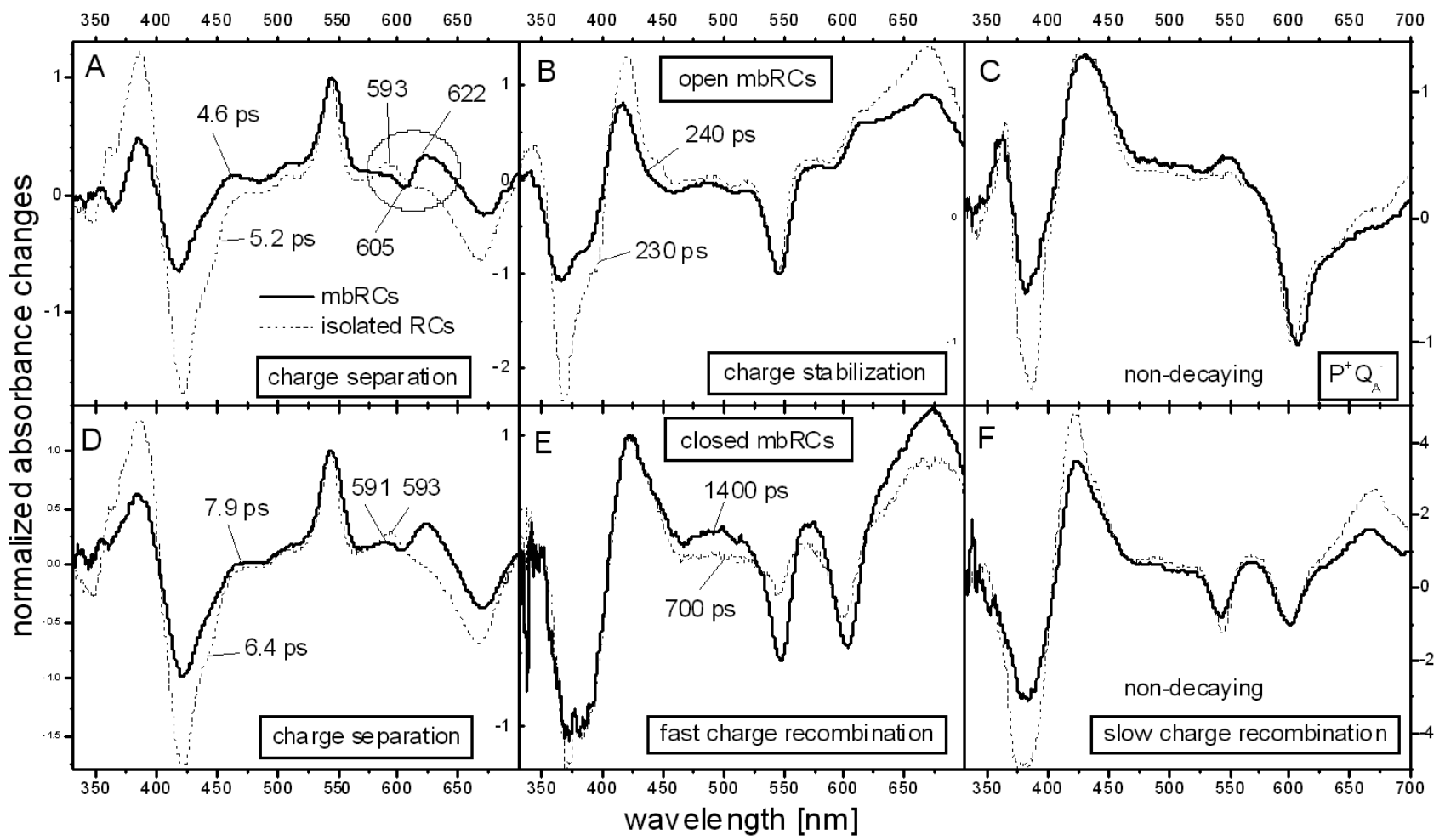

Fig. 4. Comparison of DASes for mbRCs and isolated RCs from Rba. sphaeroides. (A)-(C) DASes for open RCs, (D) - (F) DASes for closed RCs.

Closer inspection of the lineshapes of particular DAS and their comparison with the lineshapes of the respective DAS for isolated RCs [6] revealed minor differences between these two types of sample (Fig. 4). For open RCs almost all the spectral features were reproduced between isolated RCs and mbRCs in all three pairs of spectra compared in Fig. 4A-C.

One small but important difference was observed in the circled region around $600 \mathrm{~nm}$ in the DAS attributed to charge separation (Fig. $4 \mathrm{~A}$ ). In the case of isolated RCs there was a clear positive band at $593 \mathrm{~nm}$, which previously has been assigned to formation of $\mathrm{B}_{\mathrm{A}}^{-}$state due to the primary charge separation intermediate reaction $\mathrm{P}^{*} \rightarrow \mathrm{P}^{+} \mathrm{B}_{\mathrm{A}}^{-}[6]$. This reaction was kinetically unresolved from the next electron transfer step from $\mathrm{B}_{\mathrm{A}}^{-}$to $\mathrm{H}_{\mathrm{A}}$ (occurring within $\approx 1 \mathrm{ps}[17]$ ). Therefore, formation of $\mathrm{H}_{\mathrm{A}}^{-}$(manifestation of which is the band at $\approx 545 \mathrm{~nm}$, Fig. $4 \mathrm{~A}$ ) and $\mathrm{B}_{\mathrm{A}}^{-}$are depicted by a single "charge separation" DAS.

In the case of isolated RCs, the band at $593 \mathrm{~nm}$ was present also in closed RCs (Fig. 4D) and its amplitude was modulated by the state of $\mathrm{Q}_{\mathrm{A}}$ (compare Fig. $4 \mathrm{~A}$ 
and 4D; see also Ref. [6]). This small band was very important in estimation of the equilibrium constant between the states $\mathrm{P}^{+} \mathrm{B}_{\mathrm{A}}^{-}$and $\mathrm{P}^{+} \mathrm{H}_{\mathrm{A}}^{-}$, and free energy gap between these two states, on the charge separation time scale [6].

In contrast to this situation for isolated RCs, in open mbRCs there was no clear positive band at $\approx 593 \mathrm{~nm}$. Instead, there was a derivative-like shape with a minimum at $605 \mathrm{~nm}$ and maximum at $622 \mathrm{~nm}$ in the spectrum of open mbRCs (Fig. 4A), and a similar feature in the equivalent spectrum of closed mbRCs (Fig. 4D). Such a shape may originate from the electrochromic blue-shift of the absorption band of a molecule absorbing in this spectral region in response to appearance of strong electrical field originating from $\mathrm{P}^{+}$and $\mathrm{H}_{\mathrm{A}}^{-}$.

An obvious candidate for this molecule is the $\mathrm{BChl} \mathrm{B}_{\mathrm{A}}$, located between $\mathrm{P}$ and $\mathrm{H}_{\mathrm{A}}$, although its $\mathrm{Q}_{\mathrm{X}}$ absorption band is closer to $600 \mathrm{~nm}$ than to $620 \mathrm{~nm}$ (Fig. 1B). A weak signature of such an electrochromic shift is also seen in isolated RCs, but a band at $593 \mathrm{~nm}$ is clearly dominating in this region (Fig. $4 \mathrm{~A}$ ). Coexistence of the spectral signatures of $\mathrm{B}_{\mathrm{A}}^{-}$formation (in a small fraction of probed $\mathrm{RCs}$ ) and an electrochromic shift of the $\mathrm{B}_{\mathrm{A}} \mathrm{Q}_{\mathrm{X}}$ band due to $\mathrm{P}^{+} \mathrm{H}_{\mathrm{A}}^{-}$formation should not be surprising providing that the charge separation spectrum represents two ET steps that are not resolved one from another. The difference between the charge separation DAS in open isolated RCs and mbRCs is interpreted by us as indicating that the equilibrium between $\mathrm{P}^{+} \mathrm{B}_{\mathrm{A}}^{-}$and $\mathrm{P}^{+} \mathrm{H}_{\mathrm{A}}^{-}$ on the charge separation time scale is more evenly distributed between these two states in the case of isolated RCs, whereas it is much more shifted towards $\mathrm{P}^{+} \mathrm{H}_{\mathrm{A}}^{-}$in the case of mbRCs (Fig. 5).

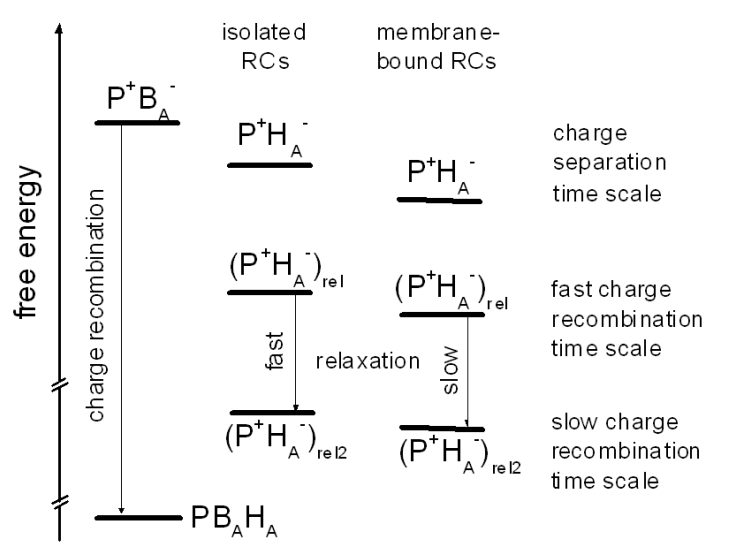

Fig. 5. Schematic model presenting similarities and differences between free energies for different states in membrane-bound and isolated reaction centers from Rba. sphaeroides. The difference in free energy between the ground $\mathrm{PB}_{\mathrm{A}} \mathrm{H}_{\mathrm{A}}$ state and all other states is under-represented (breaks in energy scale).

Inspection of the charge separation DAS in closed mbRCs (Fig. 4D) confirms the above conclusions, although apart from the electrochromic shift feature, a weak absorption band at $591 \mathrm{~nm}$ appears, similar to that at $593 \mathrm{~nm}$ in isolated RCs. This may indicate that the equilibrium between $\mathrm{P}^{+} \mathrm{B}_{\mathrm{A}}^{-}$and $\mathrm{P}^{+} \mathrm{H}_{\mathrm{A}}^{-}$shifts a little towards $\mathrm{P}^{+} \mathrm{B}_{\mathrm{A}}^{-}$upon $\mathrm{Q}_{\mathrm{A}}$ reduction in mbRCs. This effect is qualitatively similar to that one observed in isolated RCs, where the amplitude at $593 \mathrm{~nm}$ increased slightly upon RCs closure (compare Fig. 4A and 4D; see also Ref. [6]). The shift has been previously explained by the electrostatic field from $\mathrm{Q}_{\mathrm{A}}^{-}$(present only in closed $\mathrm{RCs}$ ) pushing the free energy level of $\mathrm{P}^{+} \mathrm{H}_{\mathrm{A}}^{-}$closer to that of $\mathrm{P}^{+} \mathrm{B}_{\mathrm{A}}^{-}[5,6]$. The same effect should occur in mbRCs.

The next two DAS obtained for open mbRCs were very much similar to the respective DAS for isolated RCs (Fig. 4B and C). The only clear difference between the two samples was different relative amplitudes of particular bands - the amplitudes of the blue and red parts of the spectra were larger in the case of isolated RCs (normalization was done at 545 and at $600 \mathrm{~nm}$ for the charge stabilization DAS (Fig. 4B) and ND spectrum (Fig. 4C), respectively). This effect was also observed for the charge separation (Fig. 4A, D) and charge recombination DAS (Fig. 4F), in open and closed RCs. This effect is most likely an instrumental artifact related to the spatial overlap of the pump and probe beams inside the sample. The cross-sections of the overlapping pump and probe pulses may be energetically (pump) and spectrally (probe) inhomogeneous. Thus, some parts of the sample probed by certain colors (wavelengths) composing white light may be excited more strongly than the others and, in consequence, the amplitude of the signal in different parts of the spectrum may be artificially modulated.

Both for closed isolated RCs and for closed mbRCs, a biphasic charge recombination was observed in the 1-2 ns temporal window studied (Figs. 2B, and 4EF; [6]). Due to the limited temporal window of the experiments the lifetimes of the faster phase (700 ps and 1400 ps for isolated RCs and mbRCs, respectively) may have quite a big error and the difference may be not significant. On the other hand, the non-decaying component may have a contribution from non-resolved phases of $\approx 3$ and $\approx 12 \mathrm{~ns}$ measured for both samples using a slower experimental setup with a much wider temporal window [5, 9]. For mbRCs, the amplitudes of both phases were similar to one another (see signals at $\approx 545, \approx 600$, and $\approx 665 \mathrm{~nm}$ in Fig. 2B), whereas for isolated RCs the 700 ps DAS was of much smaller amplitude than that of the ND signal (see Fig. 1D in Ref. [6]). This makes the overall charge recombination in mbRCs faster than that in isolated RCs (see Fig. 3B, C for direct comparison of $\mathrm{H}_{\mathrm{A}}^{-}$decay in the two samples). Previously it was proposed that the state $\mathrm{P}^{+} \mathrm{H}_{\mathrm{A}}^{-}$recombines to the ground state via the thermally activated state $\mathrm{P}^{+} \mathrm{B}_{\mathrm{A}}^{-}$and that the major parameter controlling the rate of $\mathrm{P}^{+} \mathrm{H}_{\mathrm{A}}^{-} \rightarrow \mathrm{PH}_{\mathrm{A}}$ charge recombination is the free energy gap between $\mathrm{P}^{+} \mathrm{B}_{\mathrm{A}}^{-}$and $\mathrm{P}^{+} \mathrm{H}_{\mathrm{A}}^{-}[5,6,9]$ : the bigger the gap the slower charge recombination.

This dependence explains the different charge recombination lifetimes of the two phases (Fig. 5). On the other hand, the relative contributions of fast and slow charge 
recombination phases depends also on the rate of $\mathrm{P}^{+} \mathrm{H}_{\mathrm{A}}^{-}$ energetic relaxation, the process which was proposed to be responsible for the multiphasic $\mathrm{P}^{+} \mathrm{H}_{\mathrm{A}}^{-} \rightarrow \mathrm{PH}_{\mathrm{A}}$ charge recombination [8, 9] (Fig. 5). In the frame of this model, our present results suggest that on the time scale of charge recombination, the energetic relaxation of the state $\mathrm{P}^{+} \mathrm{H}_{\mathrm{A}}^{-}$is slower for mbRCs than for isolated RCs (Fig. 5). Due to this difference, the contribution of RCs quickly recombining to the ground state (within $\approx 700-1400 \mathrm{ps}$ ) is bigger for mbRCs than for isolated RCs (compare Fig. 2B in this paper and 1D in Ref. [6]).

Figure 5 summarizes our view on energetic similarities and differences between isolated RCs and mbRCs. These include (1) energetic relaxation of the state $\mathrm{P}^{+} \mathrm{H}_{\mathrm{A}}^{-}$ in time in both samples with closed RCs; (2) a larger free energy gap between $\mathrm{P}^{+} \mathrm{B}_{\mathrm{A}}^{-}$and $\mathrm{P}^{+} \mathrm{H}_{\mathrm{A}}^{-}$in the case of mbRCs on the time scale of charge separation (both in open and closed RCs) and on the time scale of fast charge recombination (if the difference between 700 and 1400 ps is significant); (3) slower relaxation of $\mathrm{P}^{+} \mathrm{H}_{\mathrm{A}}^{-}$in closed RCs in the case of mbRCs. Furthermore, in closed RCs, the levels of $\mathrm{P}^{+} \mathrm{H}_{\mathrm{A}}^{-}$on the time scale of charge separation are shifted up for both samples compared to open RCs. The existence of energetic $\mathrm{P}^{+} \mathrm{H}_{\mathrm{A}}^{-}$relaxation in open $\mathrm{RCs}$ is not evidenced by the present contribution.

\section{Acknowledgments}

K.G. acknowledges financial support from the Polish government (project entitled "Electrostatic control of electron transfer in purple bacteria reaction center" no. N N202 127 437). M.R.J. acknowledges funding from the Biotechnology and Biological Sciences Research Council of the United Kingdom. The femtosecond transient absorption measurements were performed in the Center of Ultrafast Laser Spectroscopy, Adam Mickiewicz University, Poznań.

\section{References}

[1] M.R. Jones, G.J.S. Fowler, L.C.D. Gibson, G.G. Grief, J.D. Olsen, W. Crielaard, C.N. Hunter, Mol. Microbiol. 6, 1173 (1992).
[2] M.R. Jones, R.W. Visschers, R. van Grondelle, C.N. Hunter, Biochemistry 31, 4458 (1992).

[3] N.W.T. Woodbury, J.P. Allen, in: Anoxygenic Photosynthetic Bacteria, Eds. R.E. Blankenship, M.T. Madigan, C.E. Bauer, Kluwer Academic Publishers, Dordrecht 1995, p. 527.

[4] W. Zinth, J. Wachtveitl, Chem. Phys. Chem. 6, 871 (2005).

[5] K. Gibasiewicz, M. Pajzderska, J. Phys. Chem. B 112, 1858 (2008).

[6] K. Gibasiewicz, M. Pajzderska, M. Ziółek, J. Karolczak, A. Dobek, J. Phys. Chem. B 113, 11023 (2009).

[7] M. Volk, A. Ogrodnik, M.E. Michel-Beyerle, in Ref. [3], p. 595.

[8] N.W.T. Woodbury, W.W. Parson, Biochim. Biophys. Acta 767, 345 (1984).

[9] K. Gibasiewicz, M. Pajzderska, J.A. Potter, P.K. Fyfe, A. Dobek, K. Brettel, M.R. Jones, J. Phys. Chem. B 115, 13037 (2011).

[10] V.A. Shuvalov, W.W. Parson, Proc. Natl. Acad. Sci. USA 78, 957 (1981).

[11] M.R. Jones, M. Heer-Dawson, T.A. Mattioli, C.N. Hunter, B. Robert, FEBS Lett. 339, 18 (1994).

[12] A.R. Holzwarth, in: Biophysical Techniques in Photosynthesis, Eds. J. Amesz, A.J. Hoff, Kluwer Academic Publishers, Dordrecht 1996, p. 75.

[13] I.H.M. van Stokkum, D.S. Larsen, R. van Grondelle, Biochim. Biophys. Acta 1657, 82 (2004).

[14] K. Gibasiewicz, M. Pajzderska, J. Karolczak, A. Dobek, Phys. Chem. Chem. Phys. 11, 10484 (2009).

[15] N.W. Woodbury, M. Becker, D. Middendorf, W.W. Parson, Biochemistry 24, 7516 (1985).

[16] S. Wang, S. Lin, X. Lin, N.W. Woodbury, J.P. Allen, Photosynth. Res. 42, 203 (1994).

[17] W. Holzapfel, U. Finkele, W. Kaiser, D. Oesterhelt, H. Scheer, H.U. Stilz, W. Zinth, Proc. Natl. Acad. Sci. USA 87, 5168 (1990). 